\section{Molecular Recognition through Intercalation Chemistry: Immobilization of Organoclays on Piezoelectric Devices}

\section{Yongan Yan $^{\dagger}$ and Thomas Bein* \\ Department of Chemistry, Purdue University West Lafayette, Indiana 47907 \\ Received March 22, 1993 \\ Revised Manuscript Received May 24, 1993}

We report on a novel design for chemical sensors, based on the deposition of layered silicate materials ionexchanged with alkylammonium cations ("organoclays") on piezoelectric sensors, and their selectivity toward sorption of various small molecules (Figure 1). A growing interest in selective chemical sensors has prompted efforts to develop thin films with selective sorption capabilities. We have recently explored the design and sorption behavior of zeolite molecular sieve films on quartz crystal microbalances. ${ }^{1-3}$ Organoclays are interesting complements to zeolite sorbents because their sorption selectivities can be controlled to a large extent by the choice of layer charge density and structure and chain length of the alkylammonium ions. ${ }^{4}$ Different combinations of these factors will produce very different interlayer environments, containing polar mineral surfaces, hydrophobic organic chains, and paraffin-like layers. These materials have been studied as selective sorbents in general, ${ }^{5}$ in gas chromatography, ${ }^{6}$ and for toxic waste removal. ${ }^{7}$ The organoclays can offer preferential sorption of nonpolar and flat molecules.

The host chosen in this study was hectorite of approximate composition $\mathrm{Na}_{0.3}\left(\mathrm{Li}_{0.3} \mathrm{Al}_{0.01} \mathrm{Mg}_{2.67}\right) \mathrm{Si}_{4} \mathrm{O}_{10}(\mathrm{OH})_{2}$ (with some Ca content), supplied by American Colloid Co., with cation exchange capacity of 8 mequiv/ $10 \mathrm{~g}$, and charge density of $c a .1$ monovalent cation/100 $\AA^{2}{ }^{4}$ Hectorite is a layered material of the smectite family, which can be visualized as two tetrahedral silicate sheets fused to an edge-sharing octahedral sheet containing $\mathrm{Mg}$ and $\mathrm{Li}$. The degree of $\mathrm{Li}$ substitution for $\mathrm{Mg}$ determines the negative charge density on the sheets, which can be compensated by alkylammonium or other cations. The hectorite was initially treated with $0.1 \mathrm{M} \mathrm{NaCl}$ solution to ensure complete transformation to the sodium form. Impurities were removed by sedimentation for $12 \mathrm{~h}$. Intercalation was achieved by exchanging 3 times with 3 -fold excess (based on cation-exchange capacity) with tetramethylammonium (TMA) chloride, tetrapropylammonium

\footnotetext{
- Author for correspondence.

$\dagger$ Present address: YTC America Inc., 550 Via Alondra, Camarillo, CA 93012.

(1) Bein, T.; Brown, K.; Frye, C. G.; Brinker, C. J. J. Am. Chem. Soc. 1989, 111, 7640 .

(2) Bein, T.; Brown, K.; Brinker, C. J. Stud. Surf. Sci. Catal. 1989, $49,887$.

(3) (a) Yan, Y.; Bein, T. J. Phys. Chem. 1992, 96, 9387. (b) Yan, Y.; Bein, T. Chem. Mater. 1992, 4, 975. (c) Bein, T.; Brown, K.; Frye, C. G.; Brinker, C. J. U.S. Patent No. 5,151,110, Sep 1992.

(4) Barrer, R. M. Philos. Trans. R. Soc. London 1984, A311, 333.

(5) (a) Barrer, R. M.; Perry, G. S. J. Chem. Soc. 1961, 850. (b) Lee, J.-F.; Mortland, M. M.; Boyd, S. A.; Chiou, C. T. J. Chem. Soc., Faraday Trans. 1 1989, 85, 2953. (c) Lee, J.-F.; Mortland, M. M.; Chiou, C. T.; Kile, D. E.; Boyd, S. A. Clays Clay Miner. 1990, 38, 113.

(6) Lao, H.; Latieule, S.; Detellier, C. Chem. Mater. 1991, 3, 1009.

(7) (a) Boyd, S. A.; Shaobai, S.; Lee, J.-F.; Mortland, M. M. Clays Clay Miner. 1988, 36, 125. (b) Srinivasan, K. R.; Fogler, H. S. Clays Clay Miner. 1990, 38, 277. (c) Srinivasan, K. R.; Fogler, H. S. Clays Clay Miner. 1990, 38, 287.
}

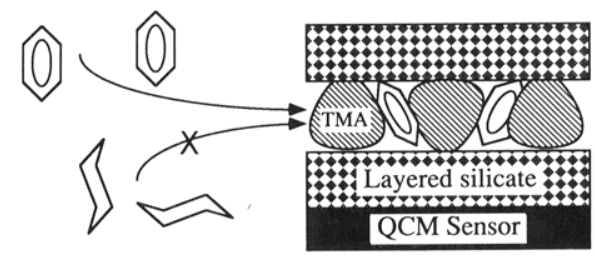

Figure 1. Schematic representation of selective benzene vs cyclohexane sorption in a TMA-hectorite film on a QCM sensor.

(TPrA) bromide, and tetrapentylammonium (TPeA) bromide (Eastman), respectively, and washing with deionized water followed by centrifugation. Intercalation of longchain organic cations (3-fold excess) was carried out three times in $30 \%$ acetonitrile/water solutions of trimethyloctadecylammonium (TrMOA) bromide, dimethyldioctadecylammonium (DMDOA) bromide, and methyltrioctadecylammonium (MTrOA) bromide (Fluka Chemicals), respectively, followed by washing in water until bromidefree.

Formation of the thin film coatings on the gold electrodes of the QCM (Fil-Tech. Inc. Boston; resonance frequency 6.0 MHz; a $1-\mathrm{Hz}$ shift corresponds to $12.1 \mathrm{ng} / \mathrm{cm}^{2}$ ) was achieved by dipping the crystals in the respective suspensions of the intercalated silicates. Depending on the dispersion properties of the organoclay, suspensions of 1 $\mathrm{mg}$ in $1 \mathrm{~mL}$ of water, $30 \%$ acetonitrile/water, or chloroform were used. The films were dried at room temperature and heated at $200^{\circ} \mathrm{C}$ in nitrogen overnight. Areal densities of the films were obtained from QCM frequency changes. Sorption isotherms of nitrogen (at liquid nitrogen temperature) and different vapors (at $25^{\circ} \mathrm{C}$ ) were taken in automated flow systems with computer-controlled adjustment of partial pressures in $\mathrm{He}$, as described previously. ${ }^{3 \mathrm{a}}$ The QCMs were degassed in a helium flow at $200^{\circ} \mathrm{C}$ for $2 \mathrm{~h}$ before isotherms were taken. Equilibrium was assumed when frequency changes were less than $1 \mathrm{~Hz}$ in $90 \mathrm{~s}$. Transient sorption of vapor pulses was measured by injecting $0.4 \mu \mathrm{L}$ of liquid into a flow system at $100^{\circ} \mathrm{C} .{ }^{3 \mathrm{a}}$

The effect of intercalating various alkylammonium cations into the hectorite host is to increase the interlayer spacing of the $\mathrm{Na}$ form ( $2.8 \AA$ when partially hydrated) to 4.2, 4.9, and 5.5 $\AA$, when TMA, TPrA, and TPeA ions are intercalated, respectively. These increased interlayer spacings are due to about one layer of hydrocarbon chains between the sheets of the clay mineral (9.6 $\AA$ thick). Intercalation of TrMOA results in an interlayer spacing of $11.1 \AA$, indicating 2-3 layers of hydrocarbon chains parallel to the clay sheets. Finally, when the areal density of octadecyl chains is doubled (dimethyldioctadexylammonium, DMDOA, $21.1 \AA$ ) or tripled (methyltrioctadecylammonium, MTrOA, $24.5 \AA$ ), they are forced to orient almost normal to the clay sheets, in a paraffinic arrangement similar to Langmuir films. The dependence of interlayer structure on hydrocarbon chain length and clay layer charge density $\left(\mathrm{eq} /(\mathrm{Si}, \mathrm{Al})_{4} \mathrm{O}_{10}\right)$ has been discussed by Lagaly. ${ }^{8}$

Figure 2 shows nitrogen sorption isotherms on QCMs for films of the organoclays described above. Sorption in

(8) Lagaly, G. Solid State Ionics $1986,22,43$. With the layer charge density of 0.3 equiv in the hectorite used here, monolayers are predicted for TMA and the other small ammonium cations studied, while TrMOA is at the borderline between bilayer and "trimolecular". Paraffin-type layer structures are predicted for DMDOA and MTrOA, when the increased number of paraffin chains per ion is considered equivalent to increasing the layer charge density. 


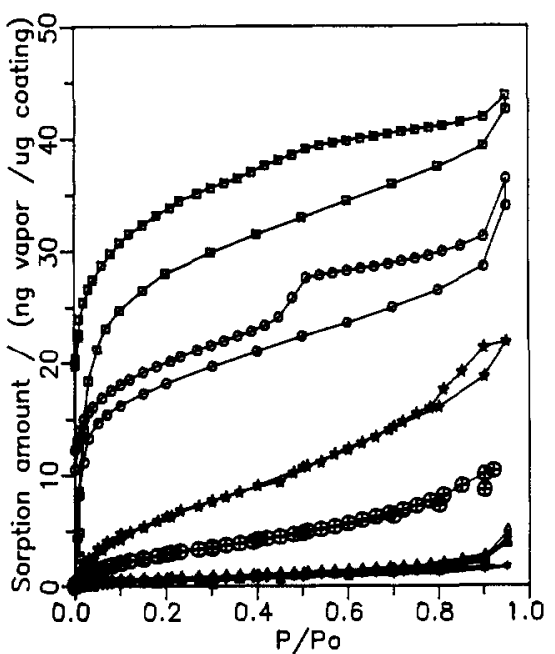

Figure 2. Nitrogen sorption isotherms at $77 \mathrm{~K}$ on films of the $\mathrm{Na}$ and different alkylammonium forms of hectorite: (口) TMA, (O) Na-form, (*) TrMOA, (+) MTrOA, (bottom three) TPrA, TPeA, and blank QCM.

the Na and TMA forms follows type I isotherms and clearly shows microporosity for nitrogen with 1.2 and 2.0 wt \% of nitrogen in the pores (derived from $t$ plots with the TPrA film as reference). The latter films show also significant external surface area ( 0.8 and $1.5 \mathrm{wt} \%$ nitrogen for monolayer coverage, respectively). In contrast, all other organoclays show type II isotherms and no microporosity (external surface areas from BET plots, in equivalent monolayer loading of nitrogen: TrMOA, $0.7 \mathrm{wt} \%$, DMDOA, 0.4 wt \%, and MTrOA, TPrA, TPeA, 0.1 wt \%, respectively). The origin of the high external surface areas of some samples may result from morphology changes after ion exchange. Electron micrographs indicate certain differences in platelet packing.

The small interlayer spacing of the $\mathrm{Na}$-hectorite film is reflected in the effective exclusion of larger hydrocarbon molecules. Figure 3A shows QCM responses to (close to saturated) vapor pulses of water, ethanol, methanol, and various hydrocarbons up to toluene and isooctane $(2,2,4-$ trimethylpentane). The pulses were less than $5 \mathrm{~s}$ long as determined with a thermal conductivity detector in series with the QCM. There is an obvious preference for water sorption, some uptake of the two alcohols, and exclusion of the hydrocarbons. The water sorption is related to hydration of the metal cations and the polar nature of the exposed silicate sheets of the clay layers. Similarly, a TMA-intercalated film shows only a minor response to the hydrocarbons (but compare equilibrium data below: the responses to vapor pulses also depend on uptake kinetics). The exposed "mineral" surface of the clay resulting from the spacing of the TMA ions (the area of TMA ions is smaller than the area per unit charge of $100 \AA^{2}$ ) is so polar that water sorption is highly favored. In striking contrast, a $\operatorname{TrMOA}$-hectorite film with $11.1 \AA$ interlayer spacing and trimolecular intercalation shows rapid and substantial uptake of toluene and $\mathrm{CH}_{2} \mathrm{Cl}_{2}$ (in addition to water and the two alcohols), but rejects $n$-pentane, $n$-hexane, $\mathrm{CCl}_{4}$, and larger hydrocarbons (Figure 3B). The uptake of water is much less than the toluene sorption, suggesting solubilization of toluene in the intercalated hydrophobic hydrocarbon layers.

Vapor sorption isotherms at low partial pressures provide more detailed information on the selective sorption
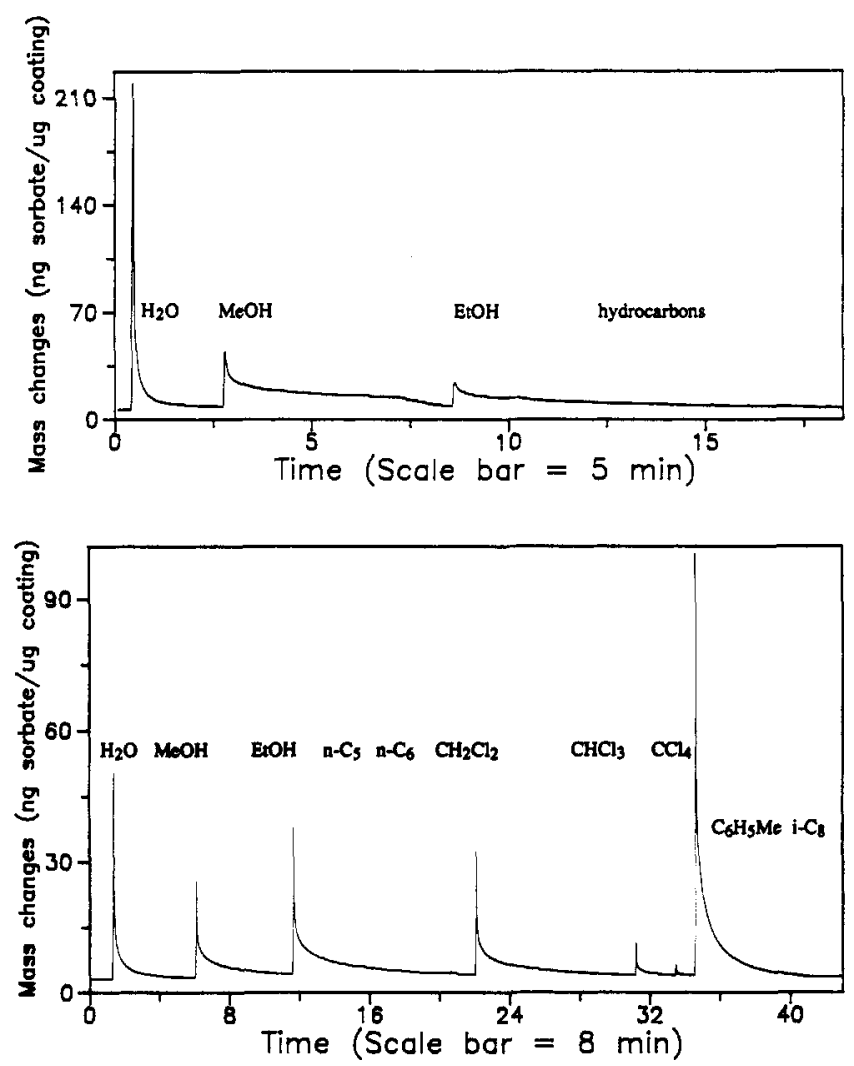

Figure 3. Transient sorption of vapor pulses at $100^{\circ} \mathrm{C}$ on 6.0 MHz QCMs coated with (A, top) Na-hectorite film $\left(52 \mu \mathrm{g} / \mathrm{cm}^{2}\right.$ ), and $(B$, bottom) coated with TrMOA-hectorite film $(181 \mu \mathrm{g} /$ $\mathrm{cm}^{2}$ ).
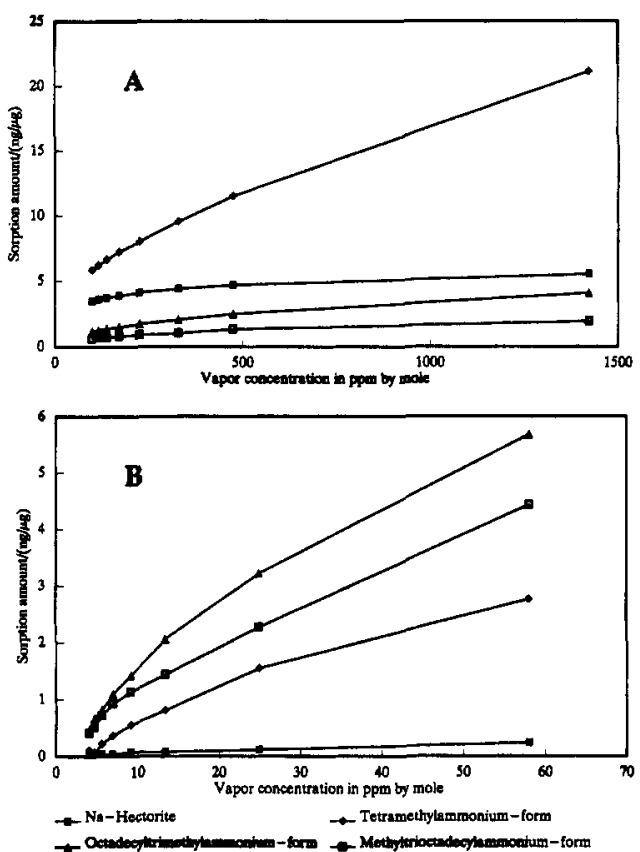

Figure 4. Vapor sorption isotherms at $25^{\circ} \mathrm{C}$ on the $\mathrm{Na}$ and different alkylammonium forms of hectorite (TMA form, $84 \mu \mathrm{g}$ / $\mathrm{cm}^{2}$; MTrOA form, $30 \mu \mathrm{g} / \mathrm{cm}^{2}$; others as in Figure 3): (A) water sorption; (B) benzene sorption.

behavior. ${ }^{9}$ Water sorption in the organoclays most closely tracks that of nitrogen (Figure 4A). The interlayer cavities

(9) The isotherms were measured at lower temperature than the pulse experiments, but selectivity trends are similar in both cases. Kinetic diameters of the relevant sorbates are as follows: nitrogen, 3.64; water, 2.65 ; cyclohesane, 6.0 ; benzene, $5.85 \mathrm{~A}$. 
produced on intercalation of TMA strongly sorb water at $25^{\circ} \mathrm{C}$. Less water is sorbed in the $\mathrm{Na}$ form and even less in the TrMOA and MTrOA forms. This behavior can be associated with the hydrophobic nature of the interlayer phase in the two latter organoclays. Thus sufficient pore size and high polarity favor water sorption in the TMA form. The $\mathrm{Na}$-hectorite apparently requires elevated temperatures to facilitate water sorption as in the pulse experiments.

A drastic reversal of the selectivities is observed when hydrocarbons are sorbed in the organoclays. Cyclohexane sorption is now most favored in the TrMOA form $(0.06 \mathrm{wt}$ $\%$ at $30 \mathrm{ppm}$ ), and in the following sequence in the other sorbent films: MTrOA (0.04) > TMA (0.02) > Na form $(0.01$ wt \%). As shown in Figure 4B, benzene is adsorbed even more strongly in the organoclays, i.e., the TrMOA film sorbs 0.36 wt $\%$, more than MTrOA (0.29), TMA $(0.18)$, and $\mathrm{Na}$-hectorite $(0.02$ wt \%) (at $30 \mathrm{ppm})$. We associate this increase over the sorption of more saturated six-rings with the compact, flat shape of benzene that should allow it to slip into the gaps between the hydrocarbon chains of both the trimolecular horizontal TrMOA structure and the paraffinic more vertical MTrOA struc- ture (relative to the clay sheets). Benzene shows also surprisingly high sorption in the TMA form. The benzene molecule can just barely be accomodated in this form when in a tilted upright orientation, as discussed by Barrer (see also Figure 1). ${ }^{\text {a }}$ It was found that benzene vapor sorption in a related TMA-montmorillonite clay was strongly favored over both $n$-heptane and cyclohexane. ${ }^{\text {ba }}$ The tight fit of benzene in the TMA clay is probably also reflected in the reduced adsorption of toluene in the same sorbent film $(0.09$ wt $\%$ at $30 \mathrm{ppm})$, while the uptake of toluene remains similar to that of benzene in the other sorbents.

In summary, this study demonstrates the potential of organically modified layered sorbents for the development of selective chemical sensors, for example, for aromatic compounds. The interplay of size exclusion and partitioning in the organic phases results in unique selectivities that can complement the molecular sieving of porous framework hosts such as zeolites.

Acknowledgment. The authors gratefully acknowledge financial support from the National Science Foundation (Division of Materials Research) and from the U.S. Department of Energy (New Mexico WERC Program). 